\title{
FMH und FMH Services an der IFAS 2012
}

\section{IFAS 2012}

www.ifas-messe.ch
Wir sind auch an der 32. Fachmesse für den Gesundheitsmarkt wieder für unsere FMH-Mitglieder da und freuen uns auf jeden persönlichen Kontakt. Kom-
Am Donnerstag, 25. Oktober 2012, haben Sie Gelegenheit, an unseren sehr beliebten Workshops teilzunehmen. Bestimmt finden Sie in unserem An-

\section{Besuchen Sie unsere Workshops am Donnerstag, 25.10.2012}

men Sie an unseren Stand auf der Galerie vor der Halle 7, diskutieren Sie mit uns und lernen Sie die vielfältigen Dienstleistungen kennen, die Ihnen die FMH und die FMH Services bieten. gebot das eine oder andere Thema, das Sie besonders interessiert. Wir freuen uns auf Ihre Anmeldung über $w w w . f m h . c h \rightarrow$ Service $\rightarrow$ IFAS 2012 .

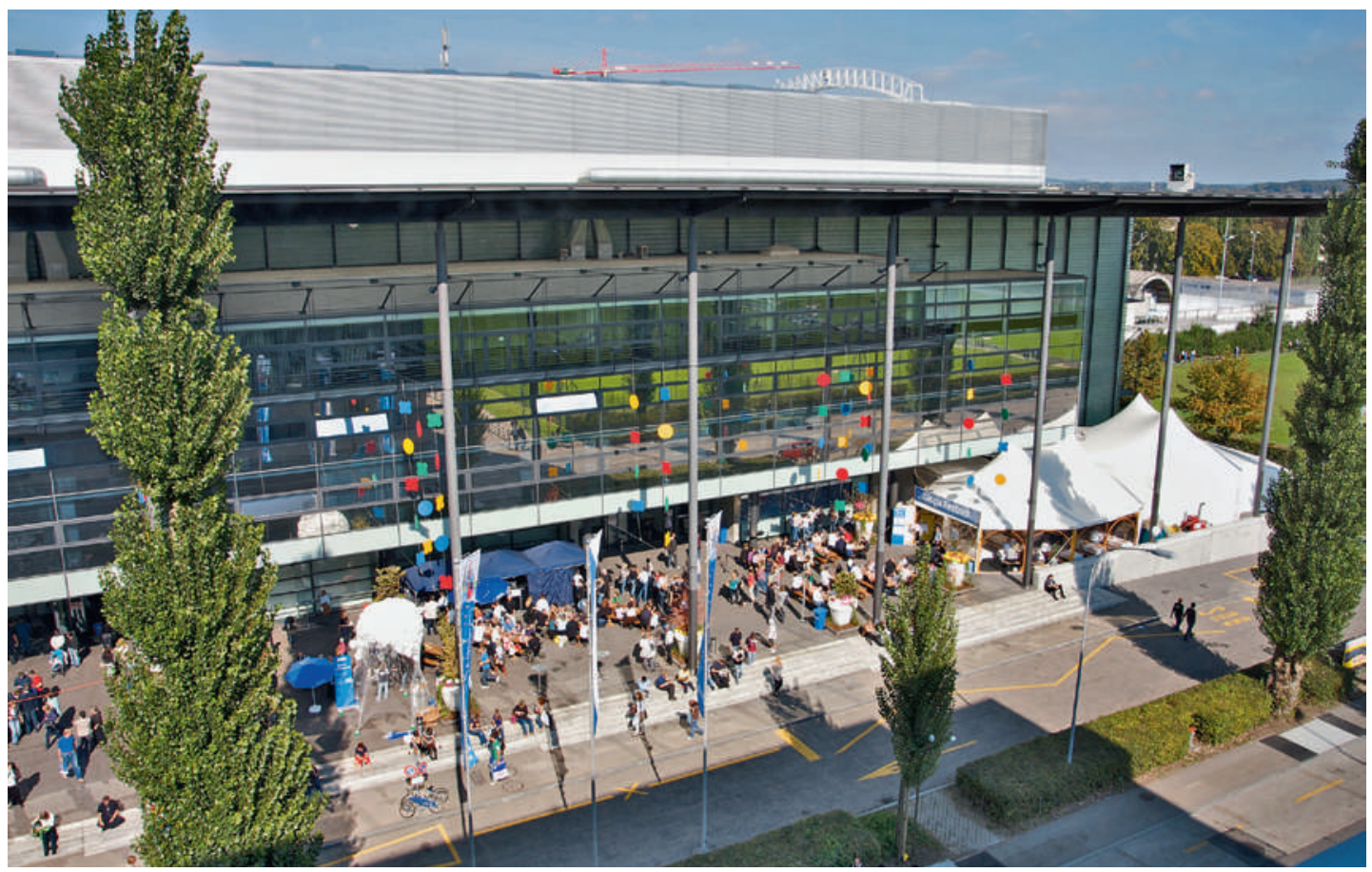


FMH-Workshop Nr. 2: Projekt Gruppenpraxis - Herausforderung für alle Beteiligten

Donnerstag, 25.10.2012, 11.30 Uhr-12.30 Uhr

Referent: Beat Bär, Leiter Beratung, FMH Consulting Services AG

Sie möchten eine Gruppenpraxis gründen oder in eine solche einsteigen. Welche Risiken und Chancen sind mit einem solchen Projekt verbunden? Wir führen Sie durch die verschiedenen Phasen der Gründung und zeigen auf, worauf beim Aufbau einer erfolgreichen Gruppenpraxis in rechtlicher, steuerlicher und betriebswirtschaftlicher Hinsicht geachtet werden muss.

FMH-Workshop Nr. 3: Einzel-/Gruppenpraxis als Einzelfirma oder als juristische Person (AG/GmbH)

Donnerstag, 25.10.2012, 13.15 Uhr-14.15 Uhr

Referenten: Rolf Willimann / Martin Brenner, FMH Treuhand Services

Soll ich als Arzt meine Praxis als Einzelfirma oder als AG/GmbH betreiben? Wie sieht es aus, wenn ich Partner in einer Praxisgemeinschaft bin? Welche Gesellschaftsform ist in diesem Fall richtig? Wir zeigen Ihnen auf der zeitlichen Achse von der Firmengründung über die Betriebsphase bis hin zum späteren Verkauf oder zu einer Liquidation, welche Aspekte bei welcher Rechtsform zu beachten sind.

\section{FMH-Workshop Nr. 4: Pensionskasse - Quo vadis?}

Donnerstag, 25.10.2012, 14.30 Uhr-15.30 Uhr

Referent: Sergio Kaufmann, FMH Insurance Services

Bei einem Grossteil der Ärzte bildet die Pensionskasse langfristig den hauptsächlichen Vermögenswert; viel Geld wird in der Pensionskasse parkiert. Jedoch setzt sich der Arzt selten mit diesem «Anlagegefäss» auseinander und ist sich wenig bewusst, wo die Chancen und Risiken liegen. Der Workshop soll diese Punkte aufzeigen und Überlegungen mitgeben, was allenfalls an der persönlichen Strategie geändert werden kann und soll.

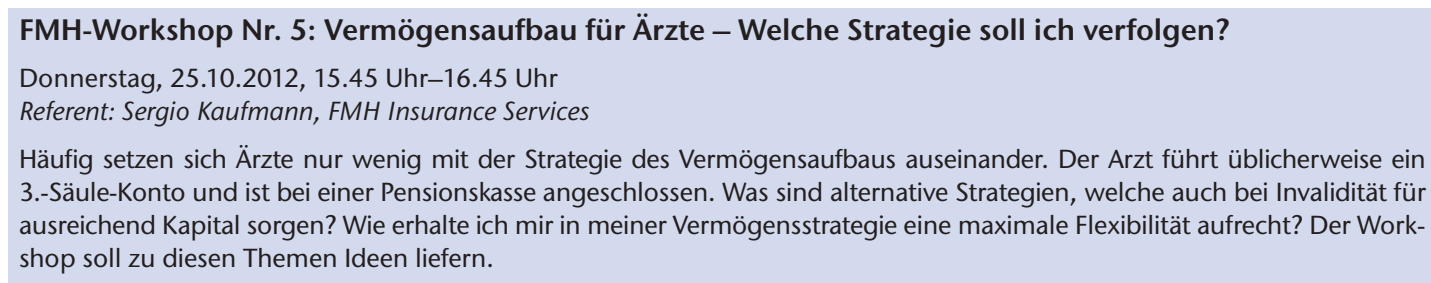

\section{Weitere Informationen}

Veranstaltungsort: Die FMH-Workshops werden durchgeführt im Seminarraum K6 (Zugang vom Galeriegeschoss vor Halle 7).

Teilnehmerkreis: Die FMH-Workshops stehen ausschliesslich Ärztinnen und Ärzten sowie deren MPAs offen (sofern die Anmeldung durch den Praxisinhaber erfolgt).

Kosten: Für FMH-Mitglieder und deren MPAs (sofern durch das FMH-Mitglied angemeldet) ist die Teilnahme an den Workshops gratis. (Nicht-FMH-Mit- glieder und deren MPAs bezahlen einen Unkostenbeitrag von 30 Franken pro Workshop.) Die Workshops werden ausschliesslich in deutscher Sprache durchgeführt.

Die Teilnehmerzahl ist begrenzt. Die Registrierung erfolgt nach Eingang der Anmeldungen. Die Teilnahmebestätigung berechtigt zum Gratiseintritt an die IFAS 2012.

\section{Anmeldung}

Online-Anmeldeformular unter:

www.fmh.ch $\rightarrow$ Service $\rightarrow$ IFAS 2012 